\title{
Photodetector device for fiber optical telecommunication systems
}

\author{
N.O. Andreyeva', V.O. Zuyev ${ }^{1}$, S.V. Morozova ${ }^{1}$, R.A. Red'ko ${ }^{1,2}$ \\ ${ }^{1}$ State University of Telecommunications, \\ 7, Solomenska str., 03680 Kyiv, Ukraine \\ ${ }^{2}$ V. Lashkaryov Institute of Semiconductor Physics, National Academy of Sciences of Ukraine, \\ 45, prospect Nauky, 03680 Kyiv, Ukraine
}

\begin{abstract}
The properties of a photodetector device with a balanced photodetector, which includes an avalanche silicon photodiode with the Schottky barrier, have been analyzed in this work. Considered here are advantages of the analyzed semiconductor photodetectors as compared with other detectors of the optical signal, in particular, their ability to register long-wave radiation, since formation of mobile carriers in them is not related with overcoming a significant surface potential barrier. To provide a high multiplication factor of an avalanche silicon photodiode with the Schottky barrier, an approach consisted of using the latter together with a receiving optical module for fiber-optic telecommunications systems has been proposed. A spectral characteristic for the Si-Au system has been obtained, it is not inferior to a germanium photodiode with the possibility of further optimization. The frequency and noise characteristics of the device have been presented. The dynamic range and the threshold of its sensitivity have been determined. The physical equivalent circuit for the photodiode of the specified type has been presented. The frequency characteristics of the output power and the noise characteristics of the receiving optical module have been also presented. It has been determined that the sensitivity threshold is $-36 \mathrm{~dB}$ for the information transfer rate close to $5 \mathrm{Gbit} / \mathrm{s}$. Reduction of intersymbol interference has been experimentally detected, which will be especially effective in the case of propagation of a signal with the corresponding shape. The obtained characteristics totally satisfy the requirements imposed on photodetectors of this type.
\end{abstract}

Keywords: avalanche silicon photodiode with Schottky barrier, fiber optic telecommunication systems, balanced photodetector.

doi: https://doi.org/10.15407/spqeo22.01.88

PACS 73.40.Lq, 73.30.+y

Manuscript received 12.02.19; revised version received 28.02.19; accepted for publication 27.03.19; published online 30.03.19.

\section{Introduction}

Currently, much attention is paid to the development of new efficient photodetector devices (PDD) for fiber-optic telecommunication systems (FOTS) [1-4]. This is due to the fact that the range and speed of information transfer essentially depend on the quality of PDD. Currently, bulk $p$ - $n$ photodiodes (PD) and bulk avalanche PD are serially used in FOTS systems. The advantage of semiconductor photodetectors as compared to, for example, photomultipliers is their ability to detect long-wave (up to $32 \mu \mathrm{m}$ ) radiation, since formation of mobile carriers in them is not associated with overcoming a significant surface potential barrier. In [5], we proposed to use the planar silicon PDs with the Schottky barrier, which are more effective in our opinion. A feature of these photodiodes is that they can be used to shift the spectral characteristics of silicon photodiodes to the long-wave region beyond their fundamental absorption edge (lowenergy limit of sensitivity of photodiodes with $p$ - $n$ junction) [6].

Photons with the energy lower than the band gap of silicon pass through the plate and are absorbed in metal, exciting hot electrons in it. The height of the internal Schottky barrier at the metal-semiconductor interface is determined by the work function of the metal. Those of the excited electrons, which have sufficient momentum in the direction to the barrier, transfer to the space charge region adjacent to the boundary that is depleted by the majority carriers (in the case of avalanche photodiodes, avalanche multiplication can take place here). The shortwave limit of sensitivity of such a device is defined by the onset of its fundamental absorption in a semiconductor (silicon), and the long-wave boundary is defined by the height of the metal-semiconductor potential barrier defined by the difference in the work 
functions of the contacting semiconductor and metal (gold, palladium or others). One of the advantages of this type of $\mathrm{PD}$ is their compatibility with the planar technology of manufacturing the counting device amplifiers. Analyzed in this work are the properties of PDD for systems with coherent coupling (SCC), which includes an avalanche PD with the Schottky barrier.

\section{Experimental and results}

It is known that avalanche photodiodes with such advantages as high sensitivity, small dimensions and low supply voltage also have disadvantages that limit their widespread use. These include temperature and temporal instability, the dependence of sensitivity on the level of background illumination, high requirements to stability of the supply voltage, as well as technological limitations that include the presence of local areas in the region of the $p-n$ junction, in which avalanche multiplication occurs earlier than in the rest value of $p$ - $n$ junction. It leads to the fact that the high multiplication factor of the photodiode is practically not realized. To solve this problem, one used various technological and structural methods.

The approach proposed in this paper consists in using an avalanche photodiode with the Schottky barrier in conjunction with a receiving optical module for fiberoptic telecommunication systems. The structure consisting of $p$-type silicon with $\mathrm{Au}$ was investigated, the surface barrier was $0.34 \mathrm{~V}$. Fig. 1 shows the spectral characteristics of PD for the Si-Au system. It can be seen that the photosensitivity extends down to $\lambda=2.5 \mu \mathrm{m}$, which makes it possible to use the device in FOTS. Indeed, at present, the wavelength preferred for transmitting optical information is $\lambda=1.55 \mu \mathrm{m}[6,7]$. It should be noted that the literature data indicate that the spectral characteristic of the diode under study in this case is not inferior to that of germanium PD [6], and in our case it is possible to further optimize PD by improving the device case.

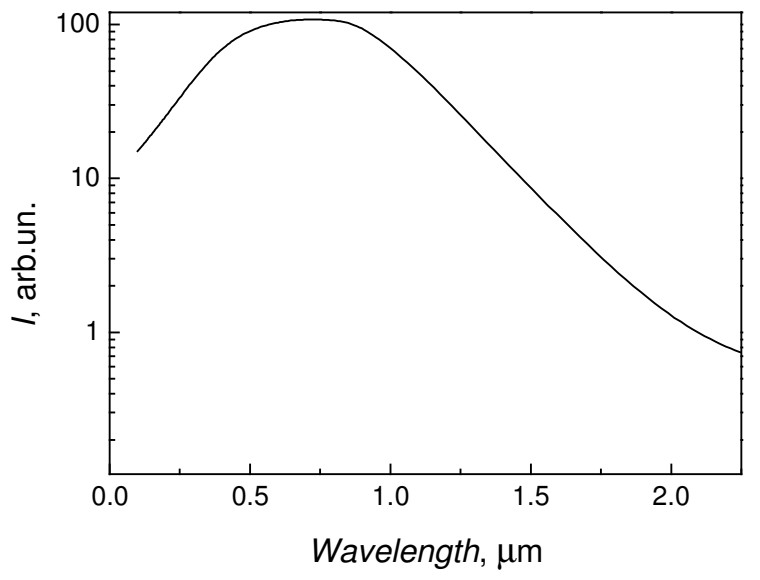

Fig. 1. Spectral characteristic of the $\mathrm{Si}-\mathrm{Au}$ system.

\subsection{Structure and characteristics of PDD for the systems with coherent coupling}

Fig. 2 shows the physical equivalent circuit of PD of the specified type. In addition to PD, an optical generator is included in PDD.

In Fig. 2, there are adopted the following notations:

$C_{p-n}$ - capacity of $p$ - $n$ transition,

$R_{l}$ - loss resistance of the photodiode,

$L_{1}$ - inductance of outputs,

$C_{C}$ - capacity of the package case,

$L_{2}$ - inductance of external outputs,

$Z_{d}$ - total resistance of the structure,

$Y_{d}-$ conductivity in the terminals of the diode,

$Z_{L}$ - load resistance,

$I_{p h}-$ photocurrent,

$I_{L}$ - current in the load.

For the most current information transfer rates (above $1 \mathrm{Gbit} / \mathrm{s}$ ), the use of a laser with distributed feedback has proved promising.

To determine the requirements for the permissible level of phase noise of a laser, the relative width of the measurement line was estimated at the half level:

$$
\Delta \mathrm{v}_{0}=\frac{\Delta \mathrm{v}_{l}}{B}
$$

where $\Delta v_{l}$ is the absolute width of the measurement line at the half level, $B$ - information transfer rate.

Fig. 3 shows the obtained dependence $\Delta v_{l}$ on $B$ for the average level of coherence used by us and an error not higher than $10^{-9}$. It is seen that the absolute width of the line grows linearly with increasing the information transfer rate.

\subsection{Principal scheme of a receiving optical module with a balanced photodetector}

Fig. 4 shows a principal scheme of a receiving optical module (ROM) for the case of the used balanced photodetector.

The differential signal of intermediate frequency falls on the input of the first stage through the correcting inductance, which increases the transfer characteristic of the photodiodes in the high-frequency

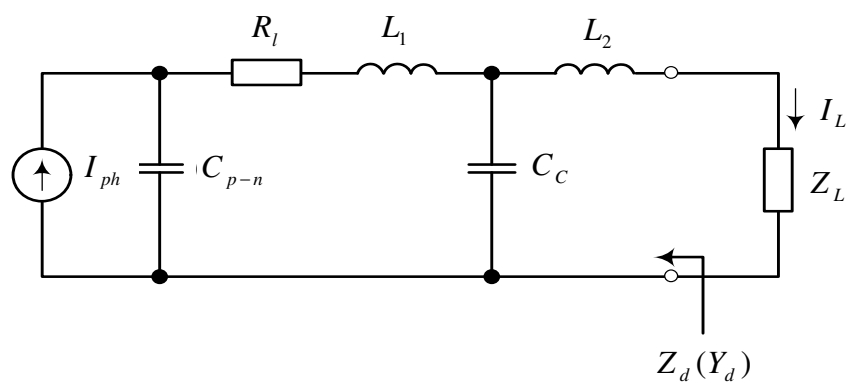

Fig. 2. Physical equivalent PD scheme with the Schottky barrier. 


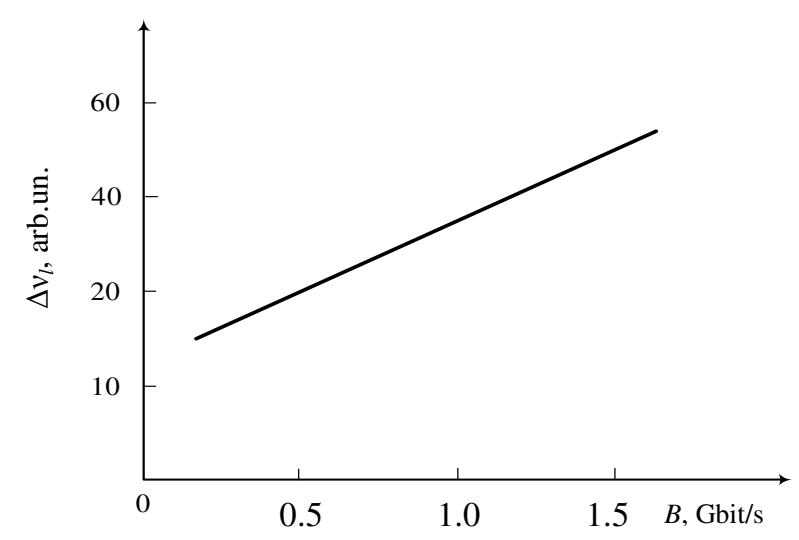

Fig. 3. Dependence of the absolute width of the measurement line at the half level of the information transfer rate.

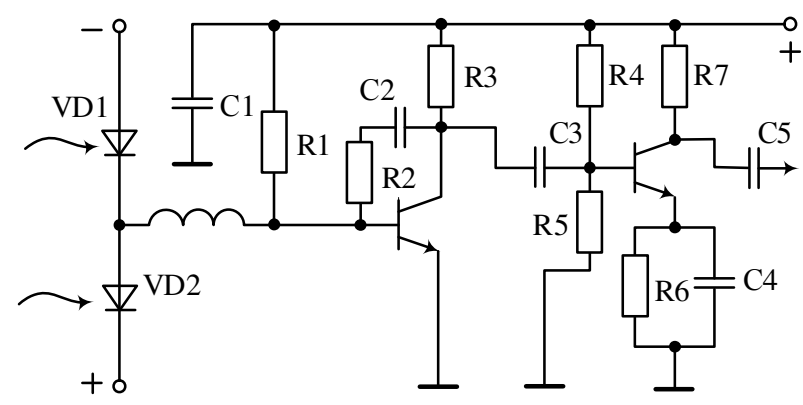

Fig. 4. Principal scheme of ROM with a balanced photodetector.

region. In the first amplifier stage, parallel feedback is applied using a serial RC circuit, and in the second one current feedback acting at low and middle frequencies.

In the absence of correlation between the sources of noise voltage and current, the minimum noise coefficient of the transistor is described by the following formula [5]:

$$
K_{n_{\min }}=1+2 R_{n} G_{y}+2 \sqrt{R_{n} G_{n}+G_{y}^{2} R_{n}^{2}}
$$

where $R_{n}$ and $G_{n}$ are the noise resistance and conductivity of the transistor, respectively, $G_{y}=1 / R$ - the real part of the conductivity of the amplifier.

\subsection{ROM characteristics at the output}

Fig. 5 shows the frequency characteristics at the ROM output and the noise characteristics. From the figure, it follows that these characteristics fully meet the requirements for PDD. In addition, it should be noted an increase in the dynamic range up to $30 \mathrm{~dB}$ and a satisfactory threshold of sensitivity $(-36 \mathrm{~dB})$ at an information transfer rate of $5 \mathrm{Gbit} / \mathrm{s}$.

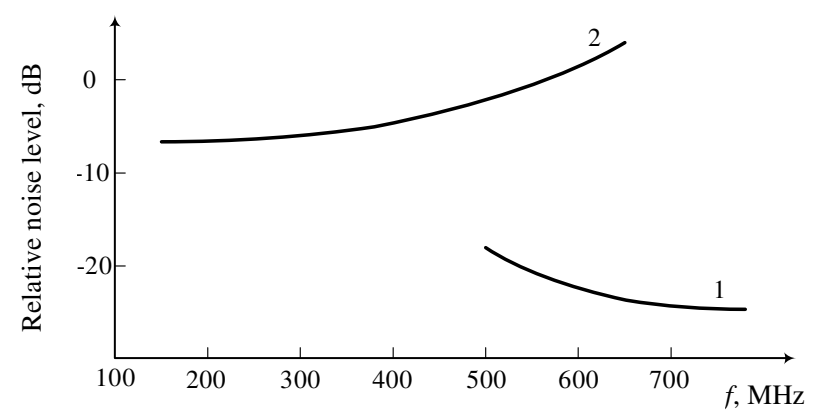

Fig. 5. Frequency characteristics of output power (1) and noise characteristics of balanced ROM (2).

It is known that the causes of noises are random thermal movement of charge carriers in conductors and semiconductor structures, their uneven distribution in bulk, etc. With increasing the temperature, the level of intrinsic noise increases. The noise intensity is quite small. However, passing through the receiver with a large gain, they create unwanted voltage at its output. The proposed PDD has a small degree of heating, which has a positive effect on its noise characteristics. Also, another important advantage of using this PDD is the experimentally observed reduction of intersymbol interference, which turns out to be especially effective in the case of signal propagation, which has a form close to the shape of the function plot $y=(\sin x) / x$.

It should be noted that each type of LED corresponds to a certain spectral characteristic, which indicates the relative sensitivity of the device to radiation of different wavelength within the spectral sensitivity of the device, and for silicon photodiodes its maximum is at $0.96 \mu \mathrm{m}$ (the operation spectral range $0.4 \ldots 1.1 \mu \mathrm{m}$ ), which imposes certain limitations on the use of the frequency range of electromagnetic waves.

The spectral characteristic of surface-barrier devices does not have a clearly pronounced maximum - in the short-wave and long-wave regions their sensitivity is correspondingly higher than that of diffusion devices. Silicon avalanche photodiodes with the Schottky barrier are sensitive in the near infrared region of the spectrum (1 to $2 \mu \mathrm{m}$ ), and with an appropriate choice of contacting metal of silicon conductivity type and operating temperature, the sensitivity region can be stretched up to $4 \mu \mathrm{m}$ or more, which makes these device structures even more promising [6].

\section{Conclusions}

The photodetector device with a balanced photodetector, which includes the silicon photodiode with the Schottky barrier, have been developed and analyzed. The frequency and noise characteristics of the receiving optical module have been measured. The dynamic range of PDD and its sensitivity threshold have been determined. It has been shown that these characteristics fully meet the requirements for PDD of this type [7]. 


\section{References}

1. Freeman R.L. Fiber Optic Systems for Telecommunications. Wiley-Interscience, 2002.

2. Liu J., Zhang T., Li Y., Ding L., Tao J., Wang Y., Wang Q., Fang J. Design and characterization of free-running InGaAsP single-photon detector with active-quenching technique. J. Appl. Phys. 2017. 122, No 1. P. 013104.

3. Chatterjee A., Agnihotri V.K., Khamari S.K., Porwal S., Bose A., Joshi S.C., Sharma T.K. Peculiarities of the current-voltage and capacitancevoltage characteristics of plasma etched $\mathrm{GaN}$ and their relevance to $n$-GaN Schottky photodetectors. J. Appl. Phys. 2018. 124, No 10. P. 104504.

4. Vieira M., Fernandes M., Louro P., Schwarz R., Schubert M. Optimized laser scanned photodiode (LSP) imaging transducer. physica status solidi (a). 2001. 185, No 1. P. 129-135.

5. Andreeva N.A., Zingaeva E.I., Zuev V.A., Muravov V.M. Frequency characteristics of the avalanche silicon photodiode with Schottky barrier. Telekomunikaciyni ta informaciyni tehnologii. 2016. 3. P. 61-65 (in Russian).

6. Rosencher E., Vinter B. Optoelectronics. Cambridge University Press, 2002.

7. Lehkii V.N, Galun B.V., Sankov O.V. Optoelectronic Elements and Devices for SpecialPurpose Systems. Novosibirsk, NGTU, 2011 (in Russian).

\section{Authors and CV}

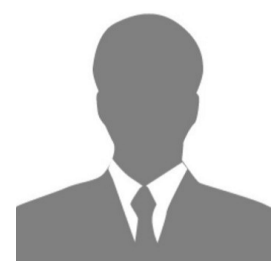

Andreeva N.O. PhD "Arms and military equipment", Associate Professor.

State University of Telecommunications

E-mail: nataliiandreeva55@ukr.net

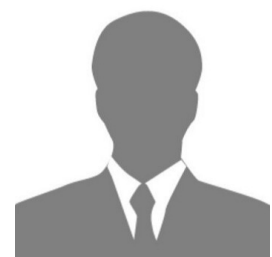

Zuyev V.O. PhD "Solid-State Physics", Dr., Professor.

State University of Telecommunications

E-mail: volodya.zyev2015@yanltx.ru

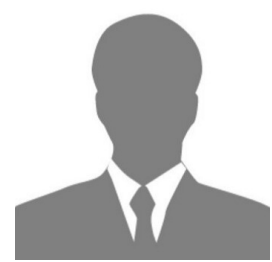

Morozova S.V.

State University of Telecommunications

E-mail: svmorozova@ukr.net.

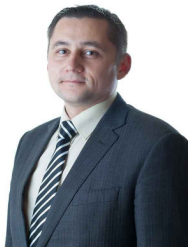

Red'ko R.A. PhD "Solid-State Physics", Senior Researcher, Associate Professor.

V. Lashkaryov Institute of Semiconductor Physics, National Academy of Sciences of Ukraine E-mail: redko.rom@gmail.com 